\title{
Effectiveness of the addition of rhythmic or discrete aiming movements training of the upper limbs on motor function and participation after stroke: protocol of a randomized controlled trial
}

\section{Renata Morales Banjai}

Universidade Cidade de Sao Paulo https://orcid.org/0000-0001-8640-779X

Leonardo Oliveira Pena Costa

Universidade Cidade de Sao Paulo

Sandra Maria Sbeghen Freitas

Universidade Cidade de Sao Paulo

Vivian Farahte Giangiardi

Universidade Cidade de Sao Paulo

Maria Liliane da Silva

Universidade Cidade de Sao Paulo

Sandra Regina Alouche ( $\nabla$ salouche@uol.com.br)

Master and Doctoral Programs in Physical Therapy, Universidade Cidade de São Paulo https://orcid.org/0000-0002-6363-0814

Study protocol

Keywords: stroke, arm, rehabilitation, exercise, physical therapy, motor control, disability

Posted Date: May 2nd, 2019

DOl: https://doi.org/10.21203/rs.2.9431/v1

License: (a) (i) This work is licensed under a Creative Commons Attribution 4.0 International License. Read Full License 


\section{Abstract}

Background Upper limb disabilities after a stroke compromise the activities of daily living. Several approaches to improve motor control and reduce disability are proposed, including specific unilateral training of the contralesional limb or bilateral training with appropriate equipment and/or robotic assistance. These approaches can be used with rhythmic or discrete movements along with usual therapy, but the most effective approach has never been determined. The objective of this study will be to verify the effect of training with rhythmic or discrete movements in conjunction with the usual therapy on motor function and participation regarding the patients' upper limbs after chronic stroke.

Methods/Design This is a 3-arm, assessor-blinded, randomized controlled trial. Seventy-five patients with chronic stroke ( $>6$ months) and upper limb paresis will be included. Participants will be randomly allocated into 3 groups: 1$)$ discrete movement training and usual therapy $(n=25), 2)$ rhythmic movement training and usual therapy $(n=25)$ and 3$)$ a control group, $(n=25)$ which will only receive the usual therapy. A five-week intervention period (10 sessions) with two sessions per week will be carried out. Outcome measures include participation, activity and motor function measured by the Stroke Impact Scale (SIS), Motor Activity Log (MAL) and Fugl-Meyer Scale, respectively; other measures are aiming movement performance, grip strength and spasticity, which will be obtained at follow-up assessments 5 weeks and 3 months after randomization. Discussion The results of this trial can support allied health professionals' decision-making processes in the treatment of motor impairment and disability after chronic stroke and reduce this condition's health-related costs.

\section{Background}

Stroke is a sudden neurological disorder that causes a diversity of impairments related to the affected brain areas. It is considered the second leading cause of death and the leading cause of disability globally [1]. Disability of the upper limbs due to stroke is common given the low rate of recovery (i.e., $15 \%$ ) in this population [2]. Stroke has various effects on body function, such as paresis, spasticity and changes of the muscle activation sequence, which entail disability [3]. These direct consequences of stroke affect activity [4] and may restrict the individual's participation [5].

Various rehabilitation methods specific to post-stroke motor impairments and disabilities have been proposed [6], including the use of specialized support apparatus and specific techniques [6, 7]. In taskoriented therapy, for example, repetitive training of daily living functional activities [8] has been suggested to promote cortical reorganization [9]. The forced unilateral training of the affected limb is used in constraint-induced movement therapy [10], [11]. The current literature states that such constraint-induced therapy induces neuroplastic cortical changes when applied to patients with mild and moderate motor impairment [12]. Bilateral training with or without rhythmic auditory stimulus [13] stimulates simultaneous use of the limbs. It is assumed that bilateral training activates interhemispheric stimulation of similar cortical areas [14]. Additionally, therapies used in conjunction with the usual therapy include robotics, which uses an auxiliary apparatus for the upper limb movements in the various phases after stroke [15] and functional electrical stimulation, [16] successfully used for shoulder pain, control of 
spasticity and manual function improvement [17]. Virtual reality [18] and mental practice [19] are also described for improvement in motor control and disability after stroke [6].

Many of the above cited approaches use discrete movements, which are voluntary movements directed at the target body part [9]. This type of movement is characterized by an initial phase of acceleration followed by a deceleration that matches the final transport period from the hand to the target [20]. Approaches that use rhythmic movements for upper limbs, however, are less common [21]. Rhythmic movements, unlike discrete movements, do not have a static phase during their implementation [22]. Rhythmic movements involve the stretching-shortening mechanism that is likely to promote greater mechanical efficiency during movement [23]. Also, rhythmic movements can be activated by general pattern generators, spinal neuronal groups capable of generating movement without relying on higher centers [24] besides the increased amount of practice. Their effects in individuals with varying degrees of paresis and motor control abnormalities resulting from stroke were not investigated. No studies compared the additive effects of upper limb movement training with rhythmic or discrete upper limb movements after chronic stroke. Therefore, the purpose of this study is to verify the effect of the training with rhythmic or discrete movements of the upper limbs in conjunction with the usual therapy on patients' motor function and participation after chronic stroke. Specifically, we intend to investigate the effect of the additive training with rhythmic movements as a rehabilitation strategy on the outcomes of motor control and participation.

\section{Methods}

\section{Approval and registration of the study}

We commenced recruitment in May 2016 in an outpatient physiotherapy clinic. The study design, procedures and informed consent were approved by the Ethics Committee in Research (research protocol number 51497415.4.0000.0064) and were prospectively registered at clinicaltrials.gov (registration number NCT02765152).

The evaluator will be responsible for screening and selecting the participants, according to the eligibility criteria. Patients unable to provide written informed consent due to their condition were excluded. The evaluator will inform the potential participants of the entire intervention process. If the participant agrees to participate in the research, he / she shall sign the consent form. Any modification of the protocol will be reported to ClinicalTrials.gov.

\section{Eligibility criteria}

All stroke survivors admitted to the outpatient clinic of the university (in Brazil) will be considered for eligibility. Participants enrolled in the treatment program at a university physiotherapy clinic will be evaluated and invited to the study. If they agree to participate in the trial, data will be collected and stored in the study files. 
The selected participants must have a clinician's diagnosis of ischemic or hemorrhagic stroke (confirmed by computed tomography or magnetic resonance imaging) and evident unilateral motor impairment. We will include adults ( $>18$ years) in the chronic stage ( $>6$ months since ictus) who can understand simple instructions (adjusted Mini Mental State Exam score according to schooling) [25]. Participants must have preserved and asymptomatic movement amplitude in the shoulder, elbow and wrist joints of the most affected upper limb and no other neurological or musculoskeletal disorders and/or clinical instability that could prevent physical effort. Participants with a history of recent drug use to modify their neuromuscular condition, e.g., botulinum toxin, somatosensory and/or perceptual deficits that hinder the completion of tasks and language and communication impairments, will be excluded.

\section{Procedures}

This is a 3-arm, randomized controlled trial with a blinded assessor. The patients will receive information about the study and the criteria for study eligibility. If the patient is considered eligible, the assessor will collect the baseline data prior to randomization. The eligible participants will be randomly allocated into three groups: discrete movement and usual therapy, rhythmic movement and usual therapy and usual therapy alone as the control group. The assessor will not know into which treatment groups patients have been allocated. The specific training, discrete or rhythmic, will be provided in addition to the usual therapy. All participants will receive the usual therapy, consisting of mobility, strength, stretching exercises and task-oriented practice [26].

The primary outcome of our study will be participation at baseline and 5 weeks ( 10 sessions) and 3 months after randomization. The secondary outcomes will be motor function and activity. Motor function will be assessed at the baseline and after 5 weeks, and activity will be assessed at the same stages and at the 3-month follow-up. Other outcomes will be provided by the analysis of aiming movement performance (movement smoothness, reaction time, movement time and end-point variability), spasticity and grip strength at baseline and 5 weeks after randomization. The interventions and the outcome measures are explained in detail below.

Insert table 1

\section{Randomization and blinding}

A researcher who has no direct participation in the recruitment and data collection will generate a randomization schedule using Microsoft Excel. The allocation will be concealed in consecutively numbered, sealed opaque envelopes. A blind assessor will conduct the baseline assessment. A researcher who was not directly involved in the evaluation and recruitment process will open the envelopes after the baseline evaluation in the participant's presence. The physical therapists responsible for intervention will be informed of the allocation. The participants from each group will be instructed not to inform the assessor about the intervention characteristics.

\section{Interventions}


All participants will receive the usual therapy. They will attend two weekly sessions of specific training for five weeks for a total of 10 treatment sessions. There will be discontinuation of treatment in case of complications such as pain in the upper limb. When the patient reports fatigue, there will be a period of rest. Participants will be included in university clinic rehabilitation programs. Will be disclosure of treatment in university newspaper. Patients included in the trial may continue complementary treatments (eg, use of orthoses) and usual therapy. The use of medications to control spasticity will not be allowed. Possible complications with the participants, will be reported to the assessor to record the information.

The study director will oversee the trial. Screening tests, assessments and treatment sessions will be monitored every week. There is no sponsor for the study

\section{Control group}

The usual therapy standards will follow the procedures established for the control group directed to the affected upper limb. The therapy will be composed of joint mobility exercises stimulating active movement of the upper limb; stretching of spastic muscles; resistance training with manual resistance in accordance with the degree of the patient's muscular strength (prioritizing the upper limb's functional specificity); motor coordination exercises, using unilateral and bilateral functional motor tasks; and taskoriented upper limb training. Additionally, others procedures related to postural control and gait will be applied when necessary [26].

\section{Discrete movement training group}

Discrete upper limb movements in conjunction with the usual therapy will be conducted for 30 minutes in two weekly sessions. The discrete movement is characterized by being unidirectional, performed from a starting point to a pre-established target. The targets have various sizes and can be positioned in various directions and distances related to the starting position. The participants will be seated with their feet fully on the floor. The table's height will be adjusted to provide support to and facilitate proper positioning of the upper limbs. The table surface will be coated with laminated material that allows the upper limb to slide over its surface.

The therapist will guide the participants to conduct the training without the use of trunk movements to avoid compensatory trunk movements during the training. The tasks are organized according to each participant's clinical evolution and function. Participants with greater sensory-motor severity (established by the Fugl-Meyer scale [27]) will begin under the therapist's assistance. The physical therapists will adjust a cylinder of various sizes (according to evolution) at the participant's hand and when necessary will make manual contact on the elbow and wrist, thereby assisting the movement. The tasks will follow a sequence with increasing complexity, providing challenges to be overcome, such as unilateral movements followed by their contralateral counterparts, increased movement distance and variation of the targets' sizes. The training will be performed unilaterally and bilaterally, i.e., the movements will be executed by the affected upper limb in isolation and combined with the ipsilesional upper limb. The targets will be circles distributed in pre-determined positions. The target that is most central and closest 
to participant will be considered the starting point, and the other targets will be placed to the left of, right of and in line with the starting point. The participants will be instructed to perform the movement at a comfortable speed, trying to reach the center of the targets. Verbal commands will be offered, which will serve as a stimulus for the beginning of the movement, involving planning demands for task execution (appendix 1).

\section{Rhythmic movement training group}

This group will perform, in addition to the usual therapy, 30 minutes of specific training of rhythmic movements twice a week. The apparatus used for the discrete training group and the participant's positioning will be the same for this group. Thus, differences between discrete and rhythmic groups are related to the task. The rhythmic movement is the repetition of movement between two targets. Although the targets will have the same distribution pattern as for the discrete training group, the participants will repeat the movement from the starting point, reaching the target and returning to the starting point consecutively for 8 to 15 repetitions. This task will be carried out without interruption of movement among the targets until the therapist establishes a moment of rest. The number of repetitions for each task will be determined by the participant's performance, i.e., when there is an increase in the number of errors, the participant will be instructed to stop the task and return to it after a period of rest. The training will be carried out unilaterally and bilaterally as proposed for the previous group. Challenges will also be proposed through increased task demands and reduced assistance of the movement performance when appropriate. Changes of direction and distance to the target will be modified along the training process (appendix 1).

\section{Assessments}

All scales and questionnaires used to assess outcomes have shown adequate measurement properties and have been translated and adapted into Brazilian Portuguese. The study's primary outcome will be participation, and the secondary outcomes will be motor function and activity.

Participation related to the upper limb due to stroke 5 weeks after randomization will be the primary outcome. Participation will be measured with the Stroke Impact Scale (SIS) [28, 29], an instrument that characterizes the individual's functional performance under its perception post-stroke. The SIS also assesses the changes along the evolution process and patients' perceptions regarding their degree of recovery [28]. The SIS is a self-reported measure, comprising eight domains (strength, manual function, daily life activity, mobility, communication, memory, emotion and thought, participation) divided into 64 items. Each item receives a score from 1 to 5 , with the highest score considered the best outcome. The domains evaluated in this study will be upper limb muscle strength, manual function, participation and general recovery perception. An algorithm generates the overall score for each domain ( $0-100$ points) [28].

The SIS has its measurement properties tested for the Brazilian population [29]. The maximum SIS effect was observed in the communication domain. The internal SIS consistency was satisfactory, and the 
domain of lower internal consistency was emotion. The intraclass correlation coefficients ranged from 0.48 (emotion) to 0.94 (manual function). A significant correlation existed among the SIS physical domains and other scales, such as the modified Rankin Scale and Barthel Index. Version 3.0 of the SIS was used in this Brazilian study and proved to be adequate on evaluation of the stroke consequences in its diversity by presenting satisfactory internal consistency, test-retest reliability and construct validity [29].

The Fugl-Meyer scale [27, 30] is an instrument for the evaluation of sensory-motor recovery, specifically developed to analyze individuals with hemiplegia or hemiparesis due to stroke. The Fugl-Meyer scale consists of five areas: upper limb motor function (maximum score of 66 points); lower limb motor function (maximum score of 34 points); sensorial function (total score of 24 points); equilibrium (maximum score of 14 points); range of joint motion (maximum score of 44 points) and pain in joint movement (maximum score of 44 points), with a total score of 226 points. Each item is scored from 0 to 2. A score of 0 means that the individual cannot perform the test, 1 means the individual partially performs it, and 2 means he or she fully performs it [27]. This study will include upper limb motor function and the upper limb total score. Therefore, the score of 60 points will be adopted for upper limb motor function and 120 points for the total upper limb score (which evaluates, along with motor function, range of motion as well as pain and sensitivity) [31]. Woodbury et al. [31] propose a classification of upper limb motor function in accordance with the capacity for voluntary movement implementation; therefore, reflex activity was removed from the measurement.

Activity will be measured with the Motor Activity Log (MAL) [10], a structured questionnaire developed by Taub et al. [10] to assess the effects of the constraint-induced movement therapy outside the intervention environment, i.e., to assess the amount of upper limb use in everyday life. This questionnaire is composed of the affected upper limb's quantitative and qualitative component use in 30 daily life activities outside the intervention context [32]. The synthesis of the original MAL's psychometric properties was reported in a review [33], and the same were tested in a study in Brazil [34]. It is worth noting that the participants in the Brazilian study presented with various degrees of impairment as classified by the Fugl-Meyer scale. Therefore, the MAL is an appropriate measure to assess the activity and participation of patients with various disability levels.

Other measures will be provided by the analysis of upper limb performance during aiming movements. Our research group has already used this analysis in previous studies [35-37]. Participants will sit at the table on a high adjustable chair that provides full trunk support. Participants will be attached to the chair and thus stabilized with a tailored vest adjusted to the trunk, limiting compensatory trunk movements. The table positioned in front of the participant will have the proper height to support the forearms. The shoulders will be maintained in neutral position for rotation, next to the trunk, and the elbows will be flexed at $90^{\circ}$.

A 12x12-inch graphic tablet (WACOM Intuos Professional ${ }^{\circledR}$ ) and a 15 -inch Samsung ${ }^{\circledR}$ monitor will be positioned at eye level on the table. The equipment will be connected to a laptop (HP AMD Pavilion® 64), 
which will control the testing task. The researcher will remain behind the participant to not interfere in the trial, and the application room will have partial acoustic isolation to eliminate distracting stimuli.

Participants who correctly performed the pen grip test on the Fugl-Meyer scale were encouraged to perform the kinematic evaluation. Each participant will perform two blocks of 10 trials with the paretic upper limb, with rest time if necessary. The trial will consist of the aiming movement with a stylus in contact with the digitizing tablet's sensitive surface from a starting point aligned with the participants' midline. The participant will be instructed to perform the movement as quickly as possible following the imperative stimulus. This stimulus is determined by the change from white to green (during $300 \mathrm{~ms}$ ) of the target to be reached, upon which the individual starts the movement. In the case of an anticipation error (reaction time less than $100 \mathrm{~ms}$ ), omission or direction, the attempt will be repeated at the end of the block. The targets will be represented on the monitor, all with diameter of $1 \mathrm{~cm}$, placed $12 \mathrm{~cm}$ away from the starting point.

The contact point's (stylus's) trajectory on the table will be recorded at a frequency of $300 \mathrm{~Hz}$. The trials' control, data storage and analysis will be carried out a by program developed in LabView 9.0 environment (National Instruments ${ }^{\circledR}$ ). The time series in $x$ and $y$ will be filtered through a $10 \mathrm{~Hz}$ Butherworth secondorder low-pass filter. Each trial's beginning and end will be defined using $5 \%$ of the trajectory's peak velocity. The reaction time, movement time, movement smoothness and resultant variable error will be calculated. The reaction time (in milliseconds) is defined as the time between the beginning of the imperative stimulus and the beginning of the stylus movement on the surface and the movement time (in milliseconds), such as the time interval between the beginning and the end of the movement. The endpoint variability (in centimeters) is considered a measure of variability for the medial-lateral $(x)$ and anterior-posterior ( $\mathrm{y}$ ) directions. The movement smoothness (in units of movement) will be evaluated by computing the number of times that the acceleration value crosses zero (positive to negative and vice versa) [37]. The average among the trials will be computed for each dependent variable and used for statistical analysis.

A manual dynamometer (Saehan $\left.{ }^{\circledR}\right)$ will be used as a measuring instrument for grip strength. Participants will be adequately seated according to the American Occupational Therapy Society. The adopted unit of measure will be kilogram force (Kgf). The participant will be instructed to sit and press the equipment's handle with maximum force. The average grip strength computed across the 3 trials [38] will be used for statistical analysis.

The spasticity of the forearm pronators, elbow flexors and wrist will be assessed with the modified Ashworth scale, a measurement scale of 0 to 4 points. The higher the score, the greater the degree of spasticity [39].

An investigator not involved in previous procedures will make data collection and monitoring. This investigator will follow any harm situation during procedures and will have the power to determine the end of the trial. Participants may withdraw from the study for any reason at any time. The investigator 
also may withdraw participants from the study to protect their safety and/or if they are unwilling or unable to comply with required study procedures.

\section{Data Analysis}

Double-data entry will be conducted to avoid mistakes. The statistical analysis will be conducted by using an intention-to-treat approach. The data's normality will be checked by histogram inspection. Descriptive statistics will be used for the groups' characterization. The between-group differences and $95 \%$ confidence intervals will be calculated using the linear mixed models. Further analysis will not be carried out. The statistical package SPSS version 19.0 for Windows will be used for all analyses.

\section{Sample size (Sample calculation)}

A minimum clinically significant difference from 4.25 to 7.25 points, or a seven-point difference, [40] was established in the Fugl-Meyer scale score [41], with an estimated standard deviation of 11 points [42]. A power of $80 \%$ was defined along with a significance level of $5 \%$, which determined a sample of 22 participants per group. This calculation included the possible loss upon follow-up of $15 \%$. Therefore, we will recruit 25 participants per group (75 patients in total).

\section{Discussion}

In this study, we present the planning and design for a 3-arm, randomized controlled trial comparing the effects of rhythmic and discrete upper limb movement training after chronic stroke. We also seek to verify the effects of these trainings in conjunction with normal care. This study's results may introduce another effective technique for conservative treatment and could guide physiotherapists in the clinical decisionmaking process for people with chronic stroke. The results of this study will be published once the study is concluded.

It is expected that training with rhythmic movements combined with usual therapy can improve motor control and the upper limb's activity and participation after a stroke when compared to additional discrete and usual therapy practices. The stretching-shortening mechanism and the central pattern generators could explain these positive results [21]. Rhythmic movements can improve the performance of the aiming movements and facilitate muscular coupling, favoring the reduction of muscular tone and leading to the increase in the practice of exercises that can favor the development of the activity and participation [43].

Plans for investigators to communicate trial results to participants, healthcare professionals, the public, and other relevant groups will be through publication.

\section{Abbreviations}


SIS: Stroke Impact Scale; MAL: Motor Activity Log; Kgf: kilogram force; UT: usual therapy; DMTG= discrete movement therapy group; RMTG= rhythmic movement therapy group.

\section{Declarations}

\section{Ethics approval and consent to participate}

The local Ethics and Research Committee of the Universidade Cidade de São Paulo, under the number CAAE 51497415.4.0000.0064 (1.454.268), approved the study and the informed consent. All participants will assign an informed consent before starting the study. Patients unable to provide written informed consent due to their condition were excluded.

\section{Consent for publish}

Not applicable

\section{Availability of data and materials}

Not applicable

\section{Competing interests}

The authors declare that they have no competing interests.

\section{Funding}

There is no funding for the study

\section{Authors' contributions}

RMB and SRA conceived of the study. RMB, SMSFF, LOPC and SRA are responsible for designing this trial's protocol and methods. RMB, SRA, VFG and MLS helped with implementation. SRA and LOPC provided statistical expertise in the clinical trial design. All authors contributed to refinement of the study protocol and approved the final manuscript.

\section{Acknowledgements}

The authors are grateful to the contributions of Letícia Estrella Curzio and Giullia Bianca Rodrigues Setti.

\section{References}

1. Feigin VL, Barker-Collo S, Parag V, Senior H, Lawes CM, Ratnasabapathy $Y$, Glen E, group As: Auckland Stroke Outcomes Study. Part 1: Gender, stroke types, ethnicity, and functional outcomes 5 years poststroke. Neurology 2010, 75(18):1597-1607. 
2. Cauraugh $\mathrm{JH}$, Summers $\mathrm{JJ}$ : Neural plasticity and bilateral movements: A rehabilitation approach for chronic stroke. Progress in neurobiology 2005, 75(5):309-320.

3. Naghdi S, Ansari NN, Mansouri K, Hasson S: A neurophysiological and clinical study of Brunnstrom recovery stages in the upper limb following stroke. Brain injury 2010, 24(11):1372-1378.

4. Harris JE, Eng JJ: Paretic upper-limb strength best explains arm activity in people with stroke. Physical therapy 2007, 87(1):88-97.

5. Faria-Fortini I, Michaelsen SM, Cassiano JG, Teixeira-Salmela LF: Upper extremity function in stroke subjects: relationships between the international classification of functioning, disability, and health domains. Journal of hand therapy : official journal of the American Society of Hand Therapists 2011, 24(3):257-264; quiz 265.

6. Pollock A, Farmer SE, Brady MC, Langhorne P, Mead GE, Mehrholz J, van Wijck F: Interventions for improving upper limb function after stroke. The Cochrane database of systematic reviews 2014(11):CD010820.

7. Liepert J: Evidence-based therapies for upper extremity dysfunction. Current opinion in neurology 2010, 23(6):678-682.

8. Winstein CJ, Stein J, Arena R, Bates B, Cherney LR, Cramer SC, Deruyter F, Eng JJ, Fisher B, Harvey RL et al: Guidelines for Adult Stroke Rehabilitation and Recovery: A Guideline for Healthcare Professionals From the American Heart Association/American Stroke Association. Stroke 2016, 47(6):e98-e169.

9. Bayona NA, Bitensky J, Salter K, Teasell R: The role of task-specific training in rehabilitation therapies. Topics in stroke rehabilitation 2005, 12(3):58-65.

10. Taub E, Miller NE, Novack TA, Cook EW, 3rd, Fleming WC, Nepomuceno CS, Connell JS, Crago JE: Technique to improve chronic motor deficit after stroke. Archives of physical medicine and rehabilitation 1993, 74(4):347-354.

11. Page SJ, Levine P, Leonard A, Szaflarski JP, Kissela BM: Modified constraint-induced therapy in chronic stroke: results of a single-blinded randomized controlled trial. Physical therapy 2008, 88(3):333340 .

12. Sirtori V, Corbetta D, Moja L, Gatti R: Constraint-induced movement therapy for upper extremities in stroke patients. The Cochrane database of systematic reviews 2009(4):CD004433.

13. Cauraugh JH, Lodha N, Naik SK, Summers JJ: Bilateral movement training and stroke motor recovery progress: a structured review and meta-analysis. Human movement science 2010, 29(5):853-870.

14. Coupar F, Pollock A, van Wijck F, Morris J, Langhorne P: Simultaneous bilateral training for improving arm function after stroke. The Cochrane database of systematic reviews 2010(4):CD006432. 
15. Lo AC, Guarino PD, Richards LG, Haselkorn JK, Wittenberg GF, Federman DG, Ringer RJ, Wagner TH, Krebs HI, Volpe BT et al: Robot-assisted therapy for long-term upper-limb impairment after stroke. The New England journal of medicine 2010, 362(19):1772-1783.

16. Alon G, Levitt AF, McCarthy PA: Functional electrical stimulation (FES) may modify the poor prognosis of stroke survivors with severe motor loss of the upper extremity: a preliminary study. American journal of physical medicine \& rehabilitation 2008, 87(8):627-636.

17. Pomeroy VM, King L, Pollock A, Baily-Hallam A, Langhorne P: Electrostimulation for promoting recovery of movement or functional ability after stroke. The Cochrane database of systematic reviews 2006(2):CD003241.

18. Kiper P, Piron L, Turolla A, Stozek J, Tonin P: The effectiveness of reinforced feedback in virtual environment in the first 12 months after stroke. Neurologia i neurochirurgia polska 2011, 45(5):436-444.

19. Bovend'Eerdt TJ, Dawes H, Sackley C, Izadi H, Wade DT: An integrated motor imagery program to improve functional task performance in neurorehabilitation: a single-blind randomized controlled trial. Archives of physical medicine and rehabilitation 2010, 91(6):939-946.

20. Connell LA, McMahon NE, Redfern J, Watkins CL, Eng JJ: Development of a behaviour change intervention to increase upper limb exercise in stroke rehabilitation. Implementation science : IS 2015, 10:34.

21. Diserens K, Perret N, Chatelain S, Bashir S, Ruegg D, Vuadens $P$, Vingerhoets $F$ : The effect of repetitive arm cycling on post stroke spasticity and motor control: repetitive arm cycling and spasticity. Journal of the neurological sciences 2007, 253(1-2):18-24.

22. Terrier R, Forestier N, Berrigan F, Germain-Robitaille M, Lavalliere M, Teasdale N: Effect of terminal accuracy requirements on temporal gaze-hand coordination during fast discrete and reciprocal pointings. Journal of neuroengineering and rehabilitation 2011, 8:10.

23. Nichols TR, Cope TC: Cross-bridge mechanisms underlying the history-dependent properties of muscle spindles and stretch reflexes. Canadian journal of physiology and pharmacology 2004, 82(89):569-576.

24. Smits-Engelsman BC, Swinnen SP, Duysens J: The advantage of cyclic over discrete movements remains evident following changes in load and amplitude. Neuroscience letters 2006, 396(1):28-32.

25. Bertolucci PH, Brucki SM, Campacci SR, Juliano Y: [The Mini-Mental State Examination in a general population: impact of educational status]. Arquivos de neuro-psiquiatria 1994, 52(1):1-7.

26. Brauer SG, Hayward KS, Carson RG, Cresswell AG, Barker RN: The efficacy of SMART Arm training early after stroke for stroke survivors with severe upper limb disability: a protocol for a randomised controlled trial. BMC neurology 2013, 13:71. 
27. Fugl-Meyer AR, Jaasko L, Leyman I, Olsson S, Steglind S: The post-stroke hemiplegic patient. 1. a method for evaluation of physical performance. Scand J Rehabil Med 1975, 7(1):13-31.

28. Duncan PW, Wallace D, Lai SM, Johnson D, Embretson S, Laster LJ: The stroke impact scale version 2.0. Evaluation of reliability, validity, and sensitivity to change. Stroke 1999, 30(10):2131-2140.

29. Carod-Artal FJ, Coral LF, Trizotto DS, Moreira CM: The stroke impact scale 3.0: evaluation of acceptability, reliability, and validity of the Brazilian version. Stroke 2008, 39(9):2477-2484.

30. Maki T, Quagliato E, Cacho E, Paz L, Nascimento N, Inoue M, Viana M: Estudo de confiabilidade da aplicação da escala de Fugl-Meyer no Brasil. Rev bras fisioter 2006, 10(2):177-183.

31. Woodbury ML, Velozo CA, Richards LG, Duncan PW: Rasch analysis staging methodology to classify upper extremity movement impairment after stroke. Archives of physical medicine and rehabilitation 2013, 94(8):1527-1533.

32. Uswatte G, Taub E, Morris D, Vignolo M, McCulloch K: Reliability and validity of the upper-extremity Motor Activity Log-14 for measuring real-world arm use. Stroke 2005, 36(11):2493-2496.

33. Saliba VA, Magalhaes Lde C, Faria CD, Laurentino GE, Cassiano JG, Teixeira-Salmela LF: [Crosscultural adaptation and analysis of the psychometric properties of the Brazilian version of the Motor Activity Log]. Revista panamericana de salud publica = Pan American journal of public health 2011, 30(3):262-271.

34. Pereira ND, Ovando AC, Michaelsen SM, Anjos SM, Lima RC, Nascimento LR, Teixeira-Salmela LF: Motor Activity Log-Brazil: reliability and relationships with motor impairments in individuals with chronic stroke. Arquivos de neuro-psiquiatria 2012, 70(3):196-201.

35. de Paiva Silva FP, Freitas SM, Silva PV, Banjai RM, Alouche SR: Ipsilesional arm motor sequence performance after right and left hemisphere damage. Journal of motor behavior 2014, 46(6):407-414.

36. Ribeiro Coqueiro P, de Freitas SM, Assuncao e Silva CM, Alouche SR: Effects of direction and index of difficulty on aiming movements after stroke. Behavioural neurology 2014, 2014:909182.

37. Banjai RM, Freitas S, Silva FPD, Alouche SR: Individuals' perception about upper limb influence on participation after stroke: an observational study. Top Stroke Rehabil 2017:1-6.

38. Mathiowetz V, Kashman N, Volland G, Weber K, Dowe M, Rogers S: Grip and pinch strength: normative data for adults. Archives of physical medicine and rehabilitation 1985, 66(2):69-74.

39. Bohannon RW, Smith MB: Interrater reliability of a modified Ashworth scale of muscle spasticity. Physical therapy 1987, 67(2):206-207. 
40. See J, Dodakian L, Chou C, Chan V, McKenzie A, Reinkensmeyer DJ, Cramer SC: A standardized approach to the Fugl-Meyer assessment and its implications for clinical trials. Neurorehabilitation and neural repair 2013, 27(8):732-741.

41. Page SJ, Fulk GD, Boyne P: Clinically important differences for the upper-extremity Fugl-Meyer Scale in people with minimal to moderate impairment due to chronic stroke. Physical therapy 2012, 92(6):791798.

42. Page SJ, Hill V, White S: Portable upper extremity robotics is as efficacious as upper extremity rehabilitative therapy: a randomized controlled pilot trial. Clinical rehabilitation 2013, 27(6):494-503.

43. Chouhan S, Kumar S: Comparing the effects of rhythmic auditory cueing and visual cueing in acute hemiparetic stroke. International Journal of Therapy and Rehabilitation 2012, 19(6):344-351.

\section{Table 1}

Table 1. Timeline for the schedule of enrollment, interventions and assessments 


\begin{tabular}{|c|c|c|c|c|c|}
\hline Outcomes & Enrolment & $\begin{array}{l}\text { Before } \\
\text { recruitment }\end{array}$ & $\begin{array}{l}\text { Intervention } \\
\text { period (10 } \\
\text { sessions) }\end{array}$ & $\begin{array}{l}10 \text { sessions } \\
\text { follow-up } \\
\text { after } \\
\text { randomization }\end{array}$ & $\begin{array}{l}3 \text { months } \\
\text { follow-up } \\
\text { after } \\
\text { randomization }\end{array}$ \\
\hline $\begin{array}{l}\text { Eligibility } \\
\text { criteria }\end{array}$ & $\mathrm{X}$ & & & & \\
\hline $\begin{array}{l}\text { Demographic } \\
\text { data }\end{array}$ & $\mathrm{X}$ & & & & \\
\hline $\begin{array}{l}\text { Informed } \\
\text { consent }\end{array}$ & $\mathrm{x}$ & & & & \\
\hline \multicolumn{6}{|l|}{$\begin{array}{l}\text { Primary } \\
\text { outcomes }\end{array}$} \\
\hline $\begin{array}{l}\text { Participation } \\
\text { (SIS) }\end{array}$ & & $X$ & & $\mathrm{X}$ & $x$ \\
\hline \multicolumn{6}{|l|}{$\begin{array}{l}\text { Secondary } \\
\text { outcomes }\end{array}$} \\
\hline Activity (MAL) & & $x$ & & $x$ & $X$ \\
\hline $\begin{array}{l}\text { Motor function } \\
\text { (FMA) }\end{array}$ & & $x$ & & $\mathrm{x}$ & \\
\hline \multicolumn{6}{|l|}{ Other outcomes } \\
\hline $\begin{array}{l}\text { Aiming } \\
\text { movement }\end{array}$ & & $x$ & & $x$ & \\
\hline Spasticity & & $x$ & & $\mathrm{x}$ & \\
\hline Grip strength & & $x$ & & $x$ & \\
\hline \multicolumn{6}{|l|}{ Interventions } \\
\hline UT+DMTG & & & $x$ & & \\
\hline UT+RMTG & & & $x$ & & \\
\hline UT & & & $x$ & & \\
\hline
\end{tabular}

SIS= Stroke Impact Scale; MAL= Motor Activity Log; FMA= Fugl-Meyer Assessment; UT= usual therapy; $\mathrm{DMTG}=$ discrete movement therapy group; $\mathrm{RMTG}=$ rhythmic movement therapy group.

\section{Supplementary Files}

This is a list of supplementary files associated with this preprint. Click to download.

- SPIRITChecklist2018.doc 
- AppendixAPROTOCOLBMCredacted.docx

- SPIRITFillablechecklistfınal.doc 